\title{
Correlation of placental histomorphology with doppler velocimetry in preeclampsia and IUGR and their perinatal outcome
}

\author{
Divya Solipuram ${ }^{1}$, Akhila Vasudeva ${ }^{1 *}$, Prashanth Adiga $^{1}$, Ashwin Das $^{2}$, \\ Antony Sylvan D'Souza ${ }^{2}$
}

\begin{abstract}
${ }^{1}$ Department of Obstetrics and Gynecology, ${ }^{2}$ Department of Anatomy, Kasturba Medical College, Manipal Academy of Higher Education, Manipal, Karnataka, India
\end{abstract}

Received: 10 May 2021

Accepted: 03 June 2021

\section{*Correspondence:}

Dr. Akhila Vasudeva,

E-mail: akhila.vasudeva@manipal.edu

Copyright: ( $)$ the author(s), publisher and licensee Medip Academy. This is an open-access article distributed under the terms of the Creative Commons Attribution Non-Commercial License, which permits unrestricted non-commercial use, distribution, and reproduction in any medium, provided the original work is properly cited.

\begin{abstract}
Background: Though numerous placental ischemic changes are described in relation to placental insufficiency, universally accepted criteria are unavailable till date leading to under or over reporting. Present study is an attempt to define standardized grading system for placental dysmorphology and correlate it with Doppler changes. The objective was to study placental histomorphology in preeclampsia and IUGR (Intrauterine Growth Restriction), to correlate the placental histomorphology with multivessel Doppler findings and their perinatal outcome in preeclampsia and IUGR. Methods: Prospective study was done over 2 years, 64 antenatal women with preeclampsia and/or IUGR were recruited, their multivessel Doppler measurements were recorded and placental histomorphological changes were studied postdelivery which were graded as either low or high grade placentas considering degree and number of ischemic changes observed.

Results: Out of 64 cases, 33 (51.5\%) cases had low grade changes and 31 (48.5\%) had high grade changes. Out of 33 patients with low grade placentas $24(73 \%)$ had normal Doppler, $20(65 \%)$ out of 31 high grade placenta had abnormal Doppler. Placental histomorphology correlated well with Doppler abnormality (Coefficient of Kappa test). Syncytial knots $>50 \%$, presence of hypermature villi, infarcts, fibrin deposits were significantly associated with abnormal Doppler. High grade placenta group had significantly poor perinatal outcome (Chi square test).

Conclusions: We formulated a grading system of placental dysmorphology in preeclampsia and IUGR which correlated well with clinical Doppler abnormality and perinatal outcome. Further studies are warranted to develop preventive strategies aimed at specific high grade placental changes seen in pregnancies with abnormal Doppler and develop strategies to improve perinatal outcome.
\end{abstract}

Keywords: IUGR, Preeclampsia, Placental histomorphology, Perinatal outcome

\section{INTRODUCTION}

Placental insufficiency is the key pathological event in preeclampsia and IUGR (Intrauterine Growth Restriction) which are associated with increased perinatal morbidity. Many studies were done to establish underlying pathological lesions in placental insufficiency. Changes like hypermature villi, intervillous thrombi, villous edema, fetal blood vessel proliferation, fibrinoid necrosis, calcifications, fibrin deposits and increased syncytial knotting were noted in the placentas affected by preeclampsia and IUGR, though the significance of individual ischemic feature is unknown. A universally accepted classification system of placental dysfunction is unavailable. ${ }^{1}$ Placenta is a complex organ with huge reserve capacity. It can compensate for intrauterine insults and cope with stressful environment that may occur secondary to any disease/toxins, ending in good perinatal 
outcome. In case the insult exceeds placental compensatory capacity, it responds with a functional impairment. However the structural changes are initiated well in advance of functional decompensation point, making it difficult to predict the functional impairment on the grounds of structural alterations.

Placenta shows diverse responses depending on duration, degree, type, severity of insult and multiple other factors. As the response is not uniform, so are changes, making it difficult to predict the severity of insult based on structural changes. Various pathologists have tried to establish criteria for assessing the histomorphology in placental insufficiency. Despite availability of robust data on all possible histomorphological changes in placental insufficiency, consensus on the type and severity of lesion leading to placental dysfunction is lacking. ${ }^{2}$ Also these changes are very nonspecific and tend to occur even in the normal pregnancies to some degree leading to diagnostic dilemma. $^{3}$ Different descriptions are used to identify similar placental "lesions" and their grading is equally non-uniform. Present study is an attempt to overcome all the above problems and formulate a new grading system for placental dysfunction and to find its clinical correlation that could possibly help improve perinatal outcome

Abnormal doppler helps to detect those pregnancies clinically at risk, however the correlation between abnormal doppler velocimetry and the degree of placental dysmorphology is not well established. ${ }^{4}$ Hence we have attempted to study placental ischemic changes, grade their severity and correlate them with abnormal doppler and perinatal outcome.

\section{METHODS}

Our objective was to study the histomorphology of placenta in preeclampsia and IUGR and correlate it with Doppler findings and their perinatal outcome. It was a prospective observational study, conducted over two years in a tertiary care hospital. A subset of pregnant women with preeclampsia and IUGR were included. Patients were identified as Preeclampsia as per International Society of Hypertension in Pregnancy (ISSHP) criteria, and IUGR was defined as estimated fetal weight and/or abdominal circumference<10th centile for the gestation as per ultrasound..$^{5}$ Women with multiple pregnancy, chronic hypertension, congenital, chromosomal abnormalities and placenta previa were excluded. All procedures followed were in accordance with the ethical standards of the responsible committee on human experimentation (institutional and national) and Helsinki Declaration of 1975, as revised in 2008. Informed consent was obtained from all patients.

Permission from Institution Ethical Committee (IEC533/2014) was obtained. We included a subgroup of all patients who were diagnosed with preeclampsia and IUGR and fulfilled the above inclusion criteria in the study period which came to 72 . As a standard care, Doppler studies were done and they were grouped into normal Doppler group (Normal umbilical artery Doppler) and abnormal doppler group which included Raised Doppler indices, absent or reversal of end diastolic flow on umbilical artery doppler, abnormal MCA and Ductus venosus Doppler. Last doppler studies, done within 24 hours prior to delivery, were taken into account. Delivery decisions were based on maternal/fetal conditions and doppler studies as decided according to institutional protocols. Post-delivery placentas were processed as per standard procedures in the department of Anatomy for gross and histology features.
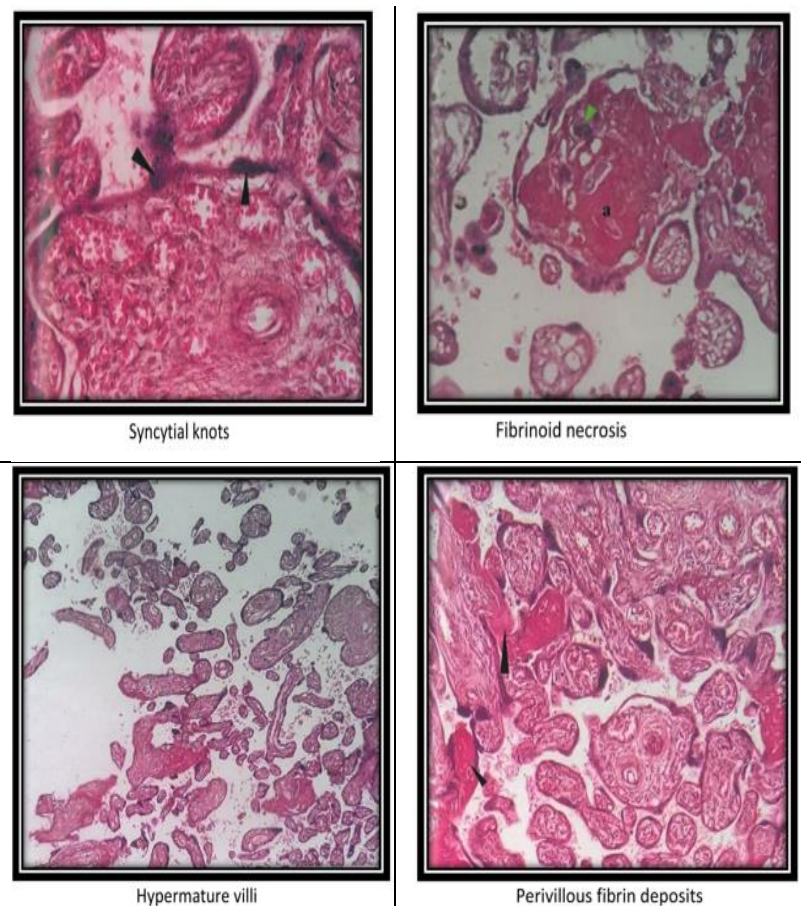

Perivillous fibrin deposits

Figure 1: Various placental histomorphology changes.

Table 1: Placental histomorphology features.

\begin{tabular}{|ll|}
\hline $\begin{array}{l}\text { Gross morphology } \\
\text { Placental weight (in } \\
\text { grams) }\end{array}$ & Histology \\
\hline Placental area (in sq cm) & Fibrinoid necrosis \\
\hline Placental volume (in cc) & $\begin{array}{l}\text { Fetal blood vessel } \\
\text { proliferation }\end{array}$ \\
\hline Fetoplacental ratio & Villous edema \\
\hline Number of infarcts & Intervillous thrombi \\
\hline & Hypermature villi \\
\hline & fibrin deposits \\
\hline & Calcifications \\
\hline
\end{tabular}

The histomorphology findings studied are shown in Table 1 and Figure 1 and Figure 2.

\section{Grading methodology of placentas}

Each of the above mentioned histomorphological features in Table 1 was divided into low grade or high grade feature considering the extent of change as in Table 2. 

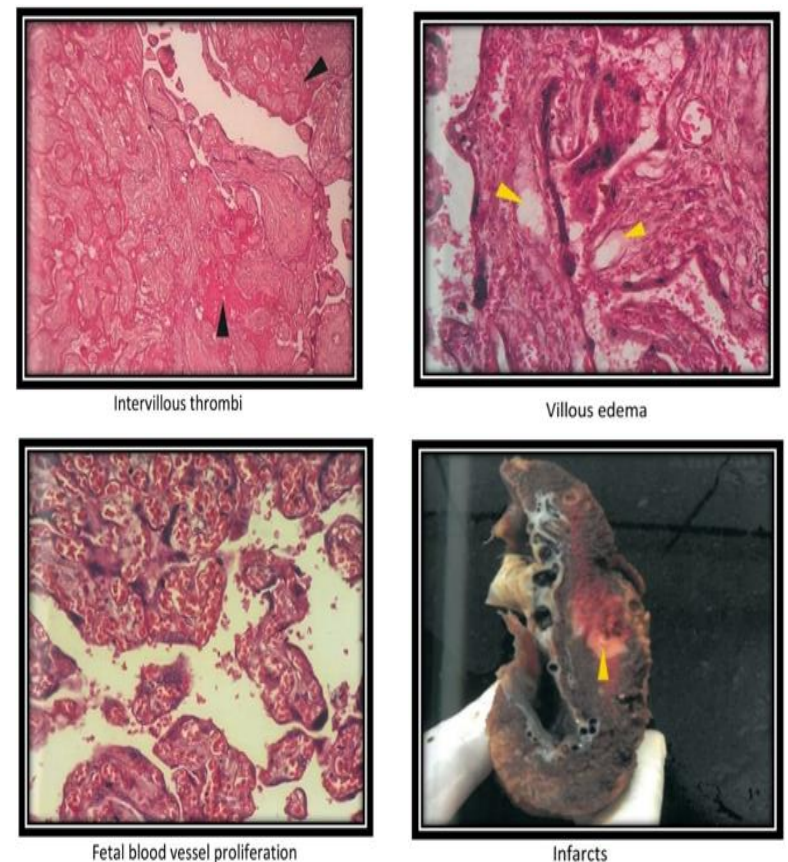

Figure 2: Various placental histomorphology changes.

Table 2: Grading of placental changes.

\begin{tabular}{|lll|}
\hline Placental change & $\begin{array}{l}\text { Low grade } \\
\text { features }\end{array}$ & $\begin{array}{l}\text { High grade } \\
\text { features }\end{array}$ \\
\hline Syncytial knots & $30-50$ & $>50$ \\
\hline Fibrinoid necrosis & $5-8$ & $>8$ \\
\hline $\begin{array}{l}\text { Fetal blood vessel } \\
\text { proliferation }\end{array}$ & $10-20$ & $\begin{array}{l}>20 \text { to massive } \\
\text { proliferation }\end{array}$ \\
\hline Villous edema & - & + \\
\hline Hypermature villi & - & + \\
\hline $\begin{array}{l}\text { Intervillous } \\
\text { thrombus }\end{array}$ & - & + \\
\hline Fibrin & - & + \\
\hline Calcification & - & + \\
\hline & $\begin{array}{l}\text { Within 2 } \\
\text { standard } \\
\text { deviation(SD) } \\
\text { for gestational } \\
\text { age(GA) }\end{array}$ & $<2$ SD for GA \\
\hline Placental weight & $<5$ & $>5$ \\
\hline Fetoplacental ratio & $\begin{array}{l}\text { Within 2 SD } \\
\text { for GA }\end{array}$ & $<2$ SD for GA \\
\hline Placental volume & $\begin{array}{l}\leq 3 \text { and } \\
\text { peripheral }\end{array}$ & $>3$ and central \\
\hline Infarcts & & \\
\hline
\end{tabular}

Histomorphological grading of placentas was considered Low grade if less than 4 high grade features were noted and high grade if 4 or more high grade features were present.

Poor perinatal outcome was defined using the outcome parameters like gestational age at delivery, birth weight, APGAR scores, duration of NICU stay, need for ventilator support. Statistical tests (Chi-square test/Coefficient of Kappa) were performed using the statistical package for social sciences (SPSS-16).

\section{RESULTS}

Out of the 72 patients initially recruited for the study, eight patients were excluded due to inability to process the placenta and totally 64 cases ( 45 preeclampsia and 19 preeclampsia with IUGR) were analyzed. Majority of patients were primigravida $(62.5 \%)$ in 3rd decade of life (61\%) and delivered preterm due to their pathology.

Table 3: Correlation between placental changes and doppler $(n=64)$.

\begin{tabular}{|c|c|c|c|}
\hline \multirow{3}{*}{$\begin{array}{l}\text { Placental } \\
\text { changes }\end{array}$} & $\begin{array}{l}\text { Normal } \\
\text { doppler }\end{array}$ & $\begin{array}{l}\text { Abnormal } \\
\text { doppler }\end{array}$ & \multirow{3}{*}{$\begin{array}{l}\mathbf{P} \\
\text { value* }\end{array}$} \\
\hline & $\mathrm{N}=35$ & $\mathbf{N}=29$ & \\
\hline & $(\%)$ & $(\%)$ & \\
\hline \multirow{2}{*}{ Low grade (33) } & 24 & 9 & \multirow{4}{*}{0.049} \\
\hline & -73 & -27 & \\
\hline \multirow{2}{*}{ High grade (31) } & 11 & 20 & \\
\hline & -35 & -65 & \\
\hline
\end{tabular}

*Coefficient of Kappa for degree of association

Table 4: Correlation of high grade placental histology with doppler $(n=64)$.

\begin{tabular}{|c|c|c|c|}
\hline \multirow{3}{*}{$\begin{array}{l}\text { High grade } \\
\text { changes }\end{array}$} & $\begin{array}{l}\text { Normal } \\
\text { doppler }\end{array}$ & $\begin{array}{l}\text { Abnormal } \\
\text { doppler }\end{array}$ & \multirow{3}{*}{$\begin{array}{l}\text { P } \\
\text { value* }\end{array}$} \\
\hline & $\mathrm{N}=35$ & $\mathrm{~N}=29$ & \\
\hline & $(\%)$ & $(\%)$ & \\
\hline \multirow{2}{*}{$\begin{array}{l}\text { Syncytial knots } \\
(\geq 50)\end{array}$} & 7 & 19 & \multirow{2}{*}{0.01} \\
\hline & -20 & -64 & \\
\hline \multirow{2}{*}{$\begin{array}{l}\text { Fibrinoid necrosis } \\
(\geq 8)\end{array}$} & 11 & 8 & \multirow{2}{*}{$>0.05$} \\
\hline & -32 & -26 & \\
\hline \multirow{2}{*}{$\begin{array}{l}\text { Fetal blood vessel } \\
\text { proliferation }(\geq 20)\end{array}$} & 4 & 6 & \multirow{2}{*}{$>0.05$} \\
\hline & -11 & -19 & \\
\hline \multirow{2}{*}{ Villous edema (+) } & 20 & 18 & \multirow{2}{*}{$>0.05$} \\
\hline & -57 & -62 & \\
\hline \multirow{2}{*}{$\begin{array}{l}\text { Hypermature villi } \\
(+)\end{array}$} & 4 & 21 & \multirow{2}{*}{0.02} \\
\hline & -12 & -72 & \\
\hline \multirow{2}{*}{$\begin{array}{l}\text { Intervillous } \\
\text { thrombus (+) }\end{array}$} & 16 & 15 & \multirow{2}{*}{$>0.05$} \\
\hline & -46 & -52 & \\
\hline \multirow{2}{*}{ Fibrin (+) } & 11 & 20 & \multirow{2}{*}{0.001} \\
\hline & -32 & -68 & \\
\hline \multirow{2}{*}{ Calcification (+) } & 18 & 15 & \multirow{2}{*}{$>0.05$} \\
\hline & -50 & -53 & \\
\hline \multirow{2}{*}{$\begin{array}{l}\text { Placental weight } \\
(<2 \text { SD) }\end{array}$} & 14 & 17 & \multirow{2}{*}{$>0.05$} \\
\hline & -40 & -60 & \\
\hline \multirow{2}{*}{$\begin{array}{l}\text { Placental area } \\
(<2 \text { SD })\end{array}$} & 15 & 17 & \multirow{2}{*}{$>0.05$} \\
\hline & -42 & -58 & \\
\hline \multirow{2}{*}{$\begin{array}{l}\text { Placental volume } \\
(<2 \text { SD) }\end{array}$} & 13 & 18 & \multirow{2}{*}{$>0.05$} \\
\hline & -37 & -63 & \\
\hline \multirow{2}{*}{$\begin{array}{l}\text { Infarcts }>3 \\
\text { and central }\end{array}$} & 7 & 23 & \multirow{2}{*}{0.03} \\
\hline & -21 & -79 & \\
\hline \multirow{2}{*}{$\begin{array}{l}\text { Fetoplacental } \\
\text { ratio }>5\end{array}$} & 14 & 17 & \multirow{2}{*}{$>0.05$} \\
\hline & -41 & -59 & \\
\hline
\end{tabular}

*Chi square test 
Out of 64 patients, 35 patients had normal doppler findings and 29 patients had abnormal Doppler findings (6 raised umbilical artery doppler, 7 abnormal MCA doppler, 12 absent end diastolic flow, 2 reversal of end diastolic flow, 2 abnormal ductus venosus doppler. Based on the grading system described in methodology, placentas of 64 patients were categorized as; Low grade placenta: 33 cases and High grade placenta: 31 cases.

Majority of the cases of low grade placenta (73\%) had normal doppler findings and $65 \%$ of the high grade placentas showed abnormal doppler and this difference was statistically significant. $(p=0.049)$ Table 3 . We correlated individual high grade placental feature with normal and abnormal doppler changes. Table 4.

Table 5: Correlation of low grade placental histology with doppler $(n=64)$.

\begin{tabular}{|c|c|c|c|}
\hline \multirow{3}{*}{$\begin{array}{l}\text { Low grade } \\
\text { changes }\end{array}$} & $\begin{array}{l}\text { Normal } \\
\text { doppler }\end{array}$ & $\begin{array}{l}\text { Abnormal } \\
\text { doppler }\end{array}$ & \multirow{3}{*}{$\begin{array}{l}\mathbf{P} \\
\text { value* }\end{array}$} \\
\hline & $\mathbf{N}=35$ & $\mathrm{~N}=\mathbf{2 9}$ & \\
\hline & $(\%)$ & $(\%)$ & \\
\hline \multirow{2}{*}{$\begin{array}{l}\text { Syncytial knots } \\
(\mathbf{3 0 - 5 0 )}\end{array}$} & 28 & 10 & \multirow{2}{*}{0.01} \\
\hline & -80 & -34 & \\
\hline \multirow{2}{*}{$\begin{array}{l}\text { Fibrinoid } \\
\text { necrosis }(5-8)\end{array}$} & 24 & 21 & \multirow{2}{*}{$>0.05$} \\
\hline & -68 & -72 & \\
\hline \multirow{2}{*}{$\begin{array}{l}\text { Fetal blood } \\
\text { vessel } \\
\text { proliferation } \\
(\mathbf{1 0 - 2 0 )}\end{array}$} & 31 & 23 & \multirow[b]{2}{*}{$>0.05$} \\
\hline & -88 & -79 & \\
\hline \multirow{2}{*}{$\begin{array}{l}\text { Villous edema } \\
(-)\end{array}$} & 15 & 11 & \multirow{2}{*}{$>0.05$} \\
\hline & -57 & -38 & \\
\hline \multirow{2}{*}{$\begin{array}{l}\text { Hypermature } \\
\text { villi (-) }\end{array}$} & 31 & 8 & \multirow{2}{*}{0.02} \\
\hline & -88 & -28 & \\
\hline \multirow{2}{*}{$\begin{array}{l}\text { Intervillous } \\
\text { thrombus (-) }\end{array}$} & 19 & 14 & \multirow{2}{*}{$>0.05$} \\
\hline & -54 & -48 & \\
\hline \multirow{2}{*}{ Fibrin (-) } & 24 & 9 & \multirow{2}{*}{0.002} \\
\hline & -68 & -32 & \\
\hline \multirow{2}{*}{$\begin{array}{l}\text { Calcification } \\
(-)\end{array}$} & 17 & 14 & \multirow{2}{*}{$>0.05$} \\
\hline & -50 & -67 & \\
\hline \multirow{2}{*}{$\begin{array}{l}\text { Placental } \\
\text { weight } \\
\text { (within } 2 \text { SD) }\end{array}$} & 21 & 12 & \multirow[b]{2}{*}{$>0.05$} \\
\hline & -60 & -40 & \\
\hline \multirow{2}{*}{$\begin{array}{l}\text { Placental area } \\
\text { (within 2SD) }\end{array}$} & 20 & 12 & \multirow{2}{*}{$>0.05$} \\
\hline & -58 & -42 & \\
\hline \multirow{2}{*}{$\begin{array}{l}\text { Placental } \\
\text { volume } \\
\text { (within } 2 \text { SD) }\end{array}$} & 22 & 11 & \multirow[b]{2}{*}{$>0.05$} \\
\hline & -63 & -37 & \\
\hline \multirow{2}{*}{$\begin{array}{l}\text { Infarcts }(<3 \\
\text { and } \\
\text { peripheral })\end{array}$} & 27 & 7 & \multirow[b]{2}{*}{$<0.05$} \\
\hline & -79 & -21 & \\
\hline \multirow{2}{*}{$\begin{array}{l}\text { Fetoplacental } \\
\text { ratio }<5\end{array}$} & 20 & 13 & \multirow{2}{*}{$>0.05$} \\
\hline & -59 & -41 & \\
\hline
\end{tabular}

*Chi square test
This sub analysis was done to see the most significant high grade feature which is closely associated with clinical manifestation of abnormal doppler. Few high grade features like syncitial knots $\geq 50$, presence of hypermature villi, fibrin deposits and infarcts were significantly associated with abnormal doppler while remaining were almost equally distributed within the two groups.

Table 6: Correlation of placental pathology with perinatal outcome.

\begin{tabular}{|c|c|c|c|}
\hline \multirow[t]{2}{*}{$\begin{array}{l}\text { Perinatal } \\
\text { outcome }\end{array}$} & $\begin{array}{l}\text { Low grade } \\
\text { placenta } \\
(33)\end{array}$ & $\begin{array}{l}\text { High } \\
\text { grade } \\
\text { placenta } \\
(31)\end{array}$ & \multirow[t]{2}{*}{$\begin{array}{l}\text { P } \\
\text { value* }^{*}\end{array}$} \\
\hline & $(\%)$ & $(\%)$ & \\
\hline \multicolumn{4}{|c|}{ Gestational age at delivery (in weeks) } \\
\hline$<32(24)$ & $7(22)$ & $17(56)$ & \multirow{3}{*}{0.01} \\
\hline $32-35(16)$ & $9(27)$ & $7(22)$ & \\
\hline$>35(24)$ & $17(51)$ & $7(22)$ & \\
\hline \multicolumn{4}{|l|}{ APGAR (1min) } \\
\hline$<9(25)$ & $9(26)$ & $16(52)$ & \multirow{2}{*}{0.166} \\
\hline $9(39)$ & $24(74)$ & $15(48)$ & \\
\hline \multicolumn{4}{|c|}{ Birth weight (in Kilograms) } \\
\hline$<1(20)$ & $8(24)$ & $12(38)$ & \multirow{3}{*}{0.02} \\
\hline $1-2(24)$ & $11(33)$ & $13(42)$ & \\
\hline$>2(20)$ & $14(43)$ & $6(20)$ & \\
\hline \multicolumn{4}{|l|}{ Need for ventilator } \\
\hline Nil/<24 hours $(30)$ & $21(63)$ & $9(30)$ & \multirow{2}{*}{0.003} \\
\hline$>24$ hours $(34)$ & $12(37)$ & $22(78)$ & \\
\hline \multicolumn{4}{|c|}{ NICU stay (in weeks) } \\
\hline$<1(20)$ & $13(40)$ & $7(23)$ & \multirow{3}{*}{0.002} \\
\hline $1-3(14)$ & $9(27)$ & $5(17)$ & \\
\hline$>3(30)$ & $11(33)$ & $19(60)$ & \\
\hline
\end{tabular}

* Chi square test

Table 5 shows association of low grade placental changes with normal/abnormal doppler findings. Only absence of hypermature villi, intervillous thrombi, infarcts $(<3$ or peripheral), 30-50 syncitial knots were associated with normal Doppler significantly. On comparing placental grades with perinatal outcome. Table 6 , patients with high grade placentas delivered at a significantly earlier gestation, had lower birth weight, required longer duration of NICU stay and ventilator support. APGAR at 1 minute however did not show significant difference between both the groups. Thus placental histomorphology correlated well with the perinatal outcome of these patients.

\section{DISCUSSION}

Many studies have proven strong association between abnormal doppler velocimetry and placental pathology, ${ }^{6}$ although significance of specific pathological lesions have not been described in these studies. A comparison is shown in Table 7. ${ }^{9,2,10}$ 
Table 7: Comparison of placental histomorphology in various studies.

\begin{tabular}{|c|c|c|c|c|c|c|c|c|}
\hline \multirow[t]{3}{*}{ Pathological change } & \multirow{2}{*}{\multicolumn{2}{|c|}{$\begin{array}{l}\text { Study } \mathrm{A}^{7} \\
\mathrm{n}=42 \\
\text { Prospective }\end{array}$}} & \multirow{2}{*}{\multicolumn{2}{|c|}{$\begin{array}{l}\text { Study } B^{2} \\
\text { n=177 } \\
\text { Prospective }\end{array}$}} & \multirow{2}{*}{\multicolumn{2}{|c|}{$\begin{array}{l}\text { Study } \mathrm{C}^{8} \\
\mathrm{n}=55 \\
\text { Prospective }\end{array}$}} & \multicolumn{2}{|c|}{ Present study } \\
\hline & & & & & & & Prosp & ective \\
\hline & $\begin{array}{l}\text { IUGR } \\
\text { (26) }\end{array}$ & $\begin{array}{l}\text { Normal } \\
(26)\end{array}$ & $\begin{array}{l}\text { Early onset } \\
\text { preeclampsia } \\
\text { (105) }\end{array}$ & $\begin{array}{l}\text { Late onset } \\
\text { preeclampsia } \\
\text { (72) }\end{array}$ & IUGR(30) & $\begin{array}{l}\text { Normal } \\
\text { (25) }\end{array}$ & $\begin{array}{l}\text { Low } \\
\text { grade }\end{array}$ & $\begin{array}{l}\text { High } \\
\text { grade }\end{array}$ \\
\hline Syncitial knots & $\begin{array}{l}\text { Not } \\
\text { studied }\end{array}$ & $\begin{array}{l}\text { Not } \\
\text { studied }\end{array}$ & $91 \%$ & $77 \%$ & $60 \%$ & $44 \%$ & $42 \%$ & $59 \%$ \\
\hline Intervillous thrombi & - & - & $21 \%$ & $12 \%$ & $\begin{array}{l}\text { Not } \\
\text { studied }\end{array}$ & $\begin{array}{l}\text { Not } \\
\text { studied }\end{array}$ & $48 \%$ & $52 \%$ \\
\hline Fibrin deposits & $62 \%$ & - & - & - & $16.7 \%$ & $1.4 \%$ & $52 \%$ & $48 \%$ \\
\hline Fibrinoid necrosis & $65 \%$ & $12 \%$ & $55 \%$ & $50 \%$ & $46.7 \%$ & $8 \%$ & $29 \%$ & $7 \%$ \\
\hline Villous edema & $46 \%$ & $27 \%$ & Not studied & Not studied & - & - & $59 \%$ & $40 \%$ \\
\hline Infarcts & $58 \%$ & $4 \%$ & $85 \%$ & $47 \%$ & $1.8 \%$ & - & - & \\
\hline Calcification & & & $59 \%$ & $51 \%$ & $60 \%$ & $50 \%$ & $51 \%$ & $48 \%$ \\
\hline
\end{tabular}

Table 8: Comparison of placental histomorphology with dopplers in various studies.

\begin{tabular}{|lllllll|}
\hline Placental features & $\begin{array}{l}\text { Salafia } \\
\text { Normal } \\
\text { doppler }\end{array}$ & $\begin{array}{l}\text { Abnormal } \\
\text { doppler }\end{array}$ & $\begin{array}{l}\text { Natalija } \\
\text { Normal } \\
\text { doppler }\end{array}$ & $\begin{array}{l}\text { Abnormal } \\
\text { doppler }\end{array}$ & $\begin{array}{l}\text { Present study } \\
\text { Normal } \\
\text { doppler }\end{array}$ & $\begin{array}{l}\text { Abnormal } \\
\text { doppler }\end{array}$ \\
\hline Intervillous thrombi & $27 \%$ & $20 \%$ & $35 \%$ & $59 \%$ & $46 \%$ & $52 \%$ \\
\hline Fibrinoid necrosis & $11.5 \%$ & $15 \%$ & $80 \%$ & $84 \%$ & $32 \%$ & $26 \%$ \\
\hline Syncytial knots & $26 \%$ & $36 \%$ & Not studied & Not studied & $20 \%$ & $60 \%$ \\
\hline Villous infarcts & $38 \%$ & $22 \%$ & $25 \%$ & $48 \%$ & $21 \%$ & $79 \%$ \\
\hline
\end{tabular}

Sub classifications of placental pathological lesions in terms of their severity leading to a grading system has not been attempted in these studies.

To overcome these issues, present study formulated a new grading system for interpretation of placental dysmorphology and found that high grade placentas were significantly associated with abnormal doppler compared to low grade placentas. Considering individual placental change, only presence of fibrin deposits, hypermature villi, syncytial knots $>50 / 100$ villi and infarcts were significantly associated with abnormal Doppler in present study. Also these high grade placentas were significantly associated with poor perinatal outcome.

In an observational study, association between umbilical artery doppler and placental histology in preeclampsia patients was studied and found that pathological changes were seen in higher proportion in abnormal doppler group compared to normal doppler group similar to our study. ${ }^{11}$ Out of the four histological features studied (syncytial knots, proliferative villous cytotrophoblast cells, thickening of subtrophoblastic basement membrane and villous hypovascularity) they found statistically significant association with villous hypovascularity and villous cytotrophoblastic proliferation which were not examined in our study $(\mathrm{p}=0.002$ and $\mathrm{p}=0.005)$. Though higher incidence of syncytial knots were noted in the abnormal doppler group in both the studies, it was statistically significant only in our study ( $\mathrm{p}=0.01 \mathrm{vs} \mathrm{p}=0.28$ ). Natalija et al categorized doppler changes as normal and abnormal doppler groups based on umbilical, middle cerebral artery and ductus venosus doppler in IUGR fetuses. ${ }^{12}$ Significantly higher incidence of villous infarcts in abnormal doppler group noted similar to our study and a higher incidence of intervillous thrombi in contrast to our study. Higher incidence of fibrinoid necrosis compared to our study; 80 and $84 \%$ in abnormal and normal doppler groups respectively vs 32 and $26 \%$ in abnormal and normal doppler groups respectively. This difference inspite of a similar clinical case selection in both the studies was likely due to differential criteria applied by various pathologists in reporting abnormal findings. Salafia et al compared incidence of placental changes like intervillous thrombi, villous infarcts, fibrinoid necrosis and syncytial knots with normal and abnormal umbilical doppler groups among IUGR patients and found no significant difference unlike our study. ${ }^{13}$ This disagreement was probably because doppler technology was not well developed those days. Table 8 shows comparison of the above 2 studies with the present study.

In a prospective study, 116 placentas were examined wherein acute atherosis with lipid laden macrophages, abnormal spiral artery remodeling, increased extravascular trophoblasts were noted in 16 placentas. ${ }^{14}$ Uterine artery doppler of these 16 cases showed abnormal uterine artery notch in $100 \%$ cases in 1st and 2nd trimesters. Remaining 100 cases with normal placental histology at delivery, $45 \%$ and $12 \%$ cases showed notch on uterine artery doppler in the first and second trimester respectively $(\mathrm{p}<0.05)$. Although our study shows similar correlation between 
abnormal umbilical artery doppler and placental histomorphology, our histopathological criteria were different and uterine artery dopplers were not studied.

In a retrospective cohort study on 100 term FGR pregnancies, 46 and 54 were in the abnormal and normal doppler groups. ${ }^{15}$ Placental pathology was more prevalent with any abnormal doppler that included umbilical, middle cerebral and uterine artery $(84.8 \%$ versus $55.6 \%, \mathrm{p}=0.002)$ which was similar to our study. Abnormal MCA Doppler had a higher prevalence of placental pathology $(96.2 \%$ versus 54.8\%, OR 20.7, 95\% CI: 2.54, 447.1, and p $<0.001)$. This further supports the higher sensitivity of MCA doppler in identifying the "true growth restricted" SGA (Small For Gestation) fetus at term. Placental weight, infarcts, syncytial knots, intervillous thrombi were studied in common by both investigators. Infarcts were significantly associated with abnormal doppler and intervillous thrombi were not which was similar in our study. However, high prevalence of syncytial knots was not associated with abnormal doppler in their study unlike ours. Studies performed in earlier decades have shown that pathological alterations in the stem villous vessels are strongly associated with vasoconstriction induced placental hypoxia and therefore evolve simultaneously with worsening umbilical artery abnormality in cases of severe FGR and early onset preeclampsia. ${ }^{16}$

Above findings reconfirm our assumption that placental lesions in combination has a better predictive value as compared to the individual placental lesion. An absolute correlation between individual placental hypoxic lesions and fetomaternal condition cannot be expected, however, when considered in a cumulative manner a better correlation was found in our study. Very few studies correlated placental histomorphology with perinatal outcome. Overall perinatal outcome variables in our study were comparable to another retrospective study. ${ }^{2}$ They categorized 117 preeclampsia cases as early (105) and late (72) onset group and studied the severity of placental changes along with uterine artery doppler abnormalities and their perinatal outcome. Perinatal outcome variables such as gestational age at delivery ( $33 \pm 3$ vs $34 \pm 2)$, Birth weight $(1798 \pm 390$ vs $1925 \pm 602)$, NICU admission $(79 \%$ vs $66.7 \%$ ), still births (3 vs 2 ), neonatal death (4 vs 7) were similar in two studies respectively. However, the association of placental pathology and perinatal outcome was not assessed in this study. A retrospective study on term IUGR fetuses, found no significant association between placental pathology and neonatal morbidity. However there was a higher prevalence of SGA fetuses in the group with placental lesions. ${ }^{15}$

A study compared placental histomorphology of 126 IUGR pregnancies and their perinatal outcome and found that 84 $(66.7 \%)$ of them had signs of placental under perfusion. Incidence of low birth weight, NICU admissions were significantly high in the placental under perfusion group similar to our study. ${ }^{17}$ However placental histomorphological grading was not used in their study. In addition, MAIN score (Morbidity Assessment Index for Newborns) was used in their study to assess perinatal outcome, which correlated well with placental under perfusion.

Currently aspirin and heparin are the only preventive strategies against placental dysfunction disorders. Villous thrombosis was directly correlated with abnormal Doppler in only one study previously which was not reproduced in other studies including ours. ${ }^{18}$ However, other placental lesions including infarcts, fibrosis, hypovascularity etc may be resulting from villous thrombosis. Recently role of reactive oxygen species and oxidative stress in the pathology behind placental infarcts have been studied. Based on this assumption, Seyithan et al in their review have suggested to appraise the role of various antioxidants in the treatment and prevention of preeclampsia. ${ }^{19}$ Further studies should focus on important unknown/unrevealed placental histomorphometric changes and pathology behind these changes that can be targeted via more effective strategies to prevent and treat placental dysfunction disorders, so that fetal vascular compromise is minimized, leading to better perinatal outcome.

Certain placental changes have recently been proven to be significantly correlated to abnormal Doppler velocimetry, including proliferative villous cytotrophoblast cells, thickening of sub-trophoblastic basement membrane and villous hypovascularity. ${ }^{11}$ We have not specifically looked into these features in our study. Also, we have not subclassified individual vessel doppler changes and their severity, hence we are unable to describe placental pathological alterations in relation to worsening doppler. Gestational age at delivery would be a confounding factor in our study, as more severe is the placental dysfunction earlier the delivery and this may affect interpretation of placental pathology.

\section{CONCLUSION}

This study graded placental histomorphological changes in preeclampsia and IUGR. High grade changes correlated well with abnormal doppler and poor perinatal outcome. In addition, certain high grade changes were strongly associated with abnormal doppler, including syncytial knots, fibrin deposits, hypermature villi and infarcts. Preventive strategies targeting these changes would help in improving the perinatal outcome considerably. Presence of villous thrombosis was not significantly associated with abnormal doppler in our study.

Funding: No funding sources

Conflict of interest: None declared

Ethical approval: The study was approved by the Institutional Ethics Committee

\section{REFERENCES}

1. Stanek J. Hypoxic patterns of placental injury. Arch Pathol Lab Med. 2013;137:706. 
2. Orabona R, Donzelli CM, Falchetti M, Santoro A, Valcamonico A, Frusca T. Placental histological patterns and uterine artery doppler velocimetry in regnancies complicated by early or late preeclampsia. Ultrasound Obstet Gynecol. 2016;47:5805.

3. Ciğercioğullari E, Filinte D, Toz E, Avciİ, Erdem B, Eminli $\dot{\mathrm{I}}$, et al. The determination of normal percentages of syncytiotrophoblastic knots in various regions of placenta: where to count the syncytial knots. Turk Patoloji Derg. 2015;31(1):1-8.

4. Vayssiere C, Sentilhes L, Eqo A, Bernard C, Cambourieu D, Flamant et al. Fetal growth restriction and intra-uterine growth res triction: guidelines for clinical practice from the French College of Gynaecologists and Obstetricians. Eur J Obstet Gynecol Reprod Biol. 2015;193:10-8.

5. Tranquilli AL, Dekker G, Magee L, Roberts J, Sibai BM, Steyn W, et al. The classification, diagnosis and management of the hypertensive disorders of pregnancy a revised statement from the ISSHP; pregnancy hypertension. Int J Women's Cardiovascular Health. 2014:4(2):97-104.

6. Goyal G, Singh KN, Agarwal R, Patel L, Khare S. Correlation of prenatal ultrasound findings with placental pathology in high risk pregnancy. J Evolution Med Dental Sci. 2014;3(03):665-72.

7. Ramadan R, Youssef H. Role of antenatal fetal doppler in correlation with histopathological, electron microscopic and immuno-histochemical findings of placentas in prediction of adverse perinatal outcome in fetal growth restricted pregnancies". EC Gynaecol. 2018;7(9):353-66.

8. Yadav S, Gupta R, Ghanghoriya S, Tripathi P. Umbilical artery colour doppler in prediction of IUGR and its correlation with histopathology of placenta. Int J Clin Obst Gynaecol. 2019;3(3):19-22.

9. Günyeli I, Erdemoğlu E, Ceylaner S, Zergeroğlu S, Mungan T. Histopathological analysis of the placental lesions in pregnancies complicated with IUGR and stillbirths in comparison with uncomplicated pregnancies. J Turkish-German Gynecol Assoc. 2011;12:75-9.
10. Kotgirwar S, Ambiye M, Athavale S, Gupta V, Trivedi S. Study of gross and histological features of placenta in intrauterine growth retardation. J Anat Soc India. 2011;60(1):37-40.

11. Gunasena GGA, Jayasundara DMC, Salgado SS, Wijesinghe PS, Biyagama B. The placenta in preeclampsia: association of histology with umbilical artery doppler velocimetry. MOJ Womens Health 2017;4(4):00092.

12. Vedmedovska N, Rezeberga D. Microscopioc lesions of placenta and doppler velocimetry related to fetal growth restriction. Arch Gynecol Obst. 2011:284(5):1087-93.

13. Salafia CM, Pezzullo JC, Minior VK, Divon MY. Placenta in absent/reversal of end diastolic flow. Reprod Biol. 1997;90:5.

14. Murat AG, Cihat F, Zerrin C. Correlation between first and second trimester uterine artery doppler velocimetry and placental bed histopathology. Int Scholarly Res Notices. 2014:890534.

15. Curtin WM, Millington KA, Ibekwe TO, Ural SH. Suspected fetal growth restriction at 37 weeks: a comparison of doppler and placental pathology. Bio Med Res Int. 2017:3723879.

16. Mifsud W, Neil J. Placental pathology in early-onset and late-onset fetal growth restriction. Fetal Diagn Ther. 2014;36:117-28.

17. Saavedra MS, Simeone S, Triunfo S, Crovetto F, Botet F. Correlation between histological signs of placental underperfusion and perinatal morbidity in late-onset small-for-gestational-age fetuses. Ultrasound Obstet Gynecol. 2015;45(2):149-55.

18. Vedmedovska N, Rezeberga D, Teibe U, Melderis I, Donders GG. Microscopioc lesions of placenta and doppler velocimetry related to fetal growth restriction. Arch Gynecol Obstet. 2011;284(5):108793.

19. Taysi S, Tascan AS, Ugur MG, Demir M. Radicals, oxidative/nitrosative stress and preeclampsia. MiniRev Med Chem. 2019;19(3):33-9.

Cite this article as: Solipuram D, Vasudeva A, Adiga P, Das A, D'Souza AS. Correlation of placental histomorphology with doppler velocimetry in preeclampsia and IUGR and their perinatal outcome. Int J Reprod Contracept Obstet Gynecol 2021;10:2811-7. 